\title{
INNOVATIONS MANAGÉRIALES ET MANAGEMENT DE PROXIMITÉ AU SEIN DES ORGANISATIONS DE SANTÉ
}

\author{
Noura Zaghmouri
}

De Boeck Supérieur | «Projectics / Proyéctica / Projectique »

2020/2 n²6 | pages 77 à 94

ISSN 2031-9703

ISBN 9782807393875

Article disponible en ligne à l'adresse :

https://www.cairn.info/revue-projectique-2020-2-page-77.htm

Distribution électronique Cairn.info pour De Boeck Supérieur.

(C) De Boeck Supérieur. Tous droits réservés pour tous pays.

La reproduction ou représentation de cet article, notamment par photocopie, n'est autorisée que dans les limites des conditions générales d'utilisation du site ou, le cas échéant, des conditions générales de la licence souscrite par votre établissement. Toute autre reproduction ou représentation, en tout ou partie, sous quelque forme et de quelque manière que ce soit, est interdite sauf accord préalable et écrit de l'éditeur, en dehors des cas prévus par la législation en vigueur en France. Il est précisé que son stockage dans une base de données est également interdit. 


\section{INNOVATIONS MANAGÉRIALES ET MANAGEMENT DE PROXIMITÉ AU SEIN DES ORGANISATIONS DE SANTÉ}

\section{Noura Zaghmouri}

Docteure en Sciences de Gestion Laboratoire Centre Européen de Recherche en Économie Financière et Gestion des Entreprises (CEREFIGE) Université de Lorraine, France

\section{RÉSUMÉ}

Les difficultés perdurant depuis plusieurs années au sein des établissements hospitaliers, imposent aux acteurs d'innover dans leurs pratiques organisationnelles et managériales. Les innovations managériales peuvent cependant être entravées par la complexité des organisations de santé et la pluralité des acteurs y exerçant. Si le manager de proximité est reconnu dans la littérature comme un acteur clé de leur succès, l'objectif de cet article est de préciser les modalités d'influence de ce professionnel sur le mécanisme de diffusion des connaissances. À partir d'une recherche-intervention menée en immersion pendant six mois au sein d'un établissement hospitalier luxembourgeois, nous accompagnons le déploiement d'une innovation managériale : le Lean Healthcare. Nos investigations révèlent l'impact du manager sur le transfert de connaissances au cours des étapes successives de l'innovation managériale, et les compétences requises telles que le leadership et l'aptitude à communiquer.

Mots clés : innovation managériale, management de proximité, organisations de santé

\section{ABSTRACT}

The difficulties that have persisted for several years in hospitals, require actors to innovate in their organizational and managerial practices. Management innovations, however, can be hampered by the complexity of health organizations and the plurality of stakeholders involved in them. If the proximity manager is recognized in literature as a key player in their success, the aim of this article is to clarify how this professional influences the mechanism of dissemination of knowledge. Based 
on a six-month immersion intervention research in a Luxembourg hospital, we support the deployment of a managerial innovation: Lean Healthcare. Our investigations reveal the impact of the manager on the transfer of knowledge during the successive stages of managerial innovation, and the skills required such as leadership and the ability to communicate.

Keywords: managerial innovation, proximity management, health organisations

\section{INTRODUCTION}

Face à la situation de crise à laquelle sont confrontés les hôpitaux (Belorgey, 2012)., la question de devoir agir ne se pose plus, elle est devenue une évidence, autant pour les acteurs politiques que pour les professionnels. Dans un contexte de restructuration du système de santé, il nous semble essentiel de rouvrir la discussion sur l'implantation des nouveaux modes d'organisation et de management des activités visant une utilisation efficiente des ressources disponibles. Nous faisons référence ici au concept d'innovation managériale qui, en se confrontant à la complexité des établissements hospitaliers, ne génère pas toujours les résultats escomptés (Lozeau, 2010).

Si les établissements de santé s'inscrivent volontiers dans une démarche d'innovation médicale (Minvielle, 2016), les travaux portant sur les innovations managériales au sein de ce contexte révèlent un essor récent et marquent la nécessité de poursuivre ces recherches au regard des défis qui se présentent pour ces organisations. Dans l'objectif d’explorer le mécanisme d'innovation managériale et les conditions favorisant leur implantation, nous nous intéressons à l'un de ses facteurs de succès : la proximité du management en considérant l'acteur associé, le manager de proximité (Kanter, 1982 ; Dubouloz, 2013). Sa position intermédiaire favorise les interactions à tous les niveaux de l'organisation et contribue ainsi à la création et la diffusion de sens (Balogun, 2010). Cependant, au sein des hôpitaux, cette position intermédiaire peut être entravée par une complexité organisationnelle et un cloisonnement limitant les interactions.

L'état de l'art actuel ne permet pas, à notre sens, d'identifier l'influence du manager de proximité sur le processus d'innovation managériale au sein des établissements hospitaliers. Nous proposons à travers cet article d'explorer cette influence à partir de l'identification des compétences requises à la diffusion des connaissances relatives à l'innovation managériale au sein de la complexité organisationnelle des hôpitaux. Nous posons la question de recherche suivante : comment le manager de proximité peut influencer la diffusion des connaissances relatives à l'innovation managériale au sein des établissements hospitaliers ? L'objectif de cet article est d'enrichir la littérature en précisant les compétences managériales soutenant le mécanisme de diffusion de connaissances inhérent au déploiement d'une innovation 
managériale au sein des hôpitaux. Il s'inscrit dans une littérature portant sur les innovations managériales au sein des hôpitaux encore naissante du fait de son caractère intangible et des difficultés à évaluer son efficacité (Bentahar \& Benzidia, 2019).

De cette recherche émergent des implications managériales portant sur une compréhension du rôle facilitateur du manager de proximité et des conditions associées permettant aux dirigeants des établissements hospitaliers de mettre en place un accompagnement efficace.

Après une présentation du cadre conceptuel se situant à la jonction des concepts d'innovation managériale, de manager de proximité et d'établissement hospitalier, nous exposons dans une seconde partie le cadre méthodologique et empirique de cette recherche. Nous décrivons ensuite, dans une troisième partie, les résultats de nos investigations et proposons enfin des pistes de discussion et les apports issus de cette recherche.

\section{CADRE CONCEPTUEL DE LA RECHERCHE}

Notre travail de recherche porte sur trois concepts interagissant les uns avec les autres : le rôle du manager de proximité au cours du processus d'innovation managériale au sein des établissements hospitaliers.

\subsection{Les innovations managériales}

Dubouloz (2014, p. 61) présente l'innovation organisationnelle en opposition à l'innovation technologique et souligne son « caractère multi-forme » couvrant « les nouvelles pratiques managériales, les nouvelles stratégies, procédures, politiques et structures organisationnelles ». Elle s'inscrit dans un concept unique intégrant les innovations organisationnelles, les innovations administratives, les innovations management et les innovations managériales, et renvoyant aux composantes organisationnelles, structurelles et managériales de ce type d'innovation (Dubouloz, 2014). La terminologie innovation managériale a été développée par Hamel et al. (2006), fondateurs de l'Innovation Management Lab à la London Business School, qui ont souhaité préciser le concept d'innovation organisationnelle en soulignant son caractère stratégique et managérial. Elle correspond à un processus « collectif, long et complexe, lié à l'apprentissage » (Dubouloz, 2013, p. 121 citant Alter, 2010 ; Scozzi et al., 2005) composé d'une succession d'étapes (Dubouloz, 2013 ; Damanpour, 1991) :

- La décision de mettre en usage : la perception d'un besoin ou problème conduit les acteurs à envisager les solutions potentielles parmi lesquelles peut émerger 
une innovation. Des échanges entre acteurs conduisent à la décision de l'appliquer.

- La mise en usage : l'expérimentation de l'innovation ainsi que sa transformation mènent à son adaptation, les acteurs de l'organisation choisissent de l'adopter ou non.

- La poursuite de l'usage : l'innovation est adoptée par les acteurs, elle constitue une routine et fait partie intégrante du fonctionnement de l'organisation. Cette étape marque le succès de l'innovation.

Dans le cadre de notre recherche, nous nous intéressons à une innovation précise : le Lean Management. Il s'agit d'une philosophie de gestion conçue dans les années 1990 à partir du système de production Toyota dans les années 1950 (Ohno, 1988). La méthode s'est développée au sein de divers secteurs, notamment le secteur des services, sous l'appellation Lean Management et vise une optimisation de la valeur perçue par le client en réduisant les pertes liées aux gaspillages dans un objectif d'excellence opérationnelle et d'application d'une démarche d'amélioration continue (Ohno, 1988). Le Lean Management génère une nouvelle organisation associée à une nouvelle philosophie et de nouvelles techniques (Womack et Jones, 2007) et est assimilé à une innovation managériale souvent citée dans les recherches sur ce concept (Aoki, 1988 ; Armbruster et al., 2008 ; Birkinshaw et al., 2008 ; Ménard, 1995 ; Niosi, 1998). Il remet en question la distribution du pouvoir décisionnel et modifie la configuration hiérarchique traditionnelle.

Dans le secteur de la santé, la terminologie Lean Management s'est adaptée sous les appellations Lean santé ou Lean healthcare, et est décrite comme une méthode efficace de réaménagement des processus (Foropon et Landry, 2014). Apparue aux Etats-Unis en 1990, l'approche s'est diffusée au Canada, en Europe, en Asie où les résultats furent quelquefois spectaculaires (Nicolay et al., 2012 ; Radnor et al., 2012). Cependant, la littérature met en exergue des résultats contrastés (Hasle, 2014). Parmi les causes d'échecs, il est à noter son application en tant que méthode cost killer visant à identifier et supprimer les sources de gaspillage sans consulter les professionnels de terrain (Kim et al., 2006 ; Deblois et Lepanto, 2013). Selon Dubouloz (2013), l'instauration d'un mode de management de proximité et la mise en place d'un processus d'apprentissage autour de l'innovation permettant sa diffusion peuvent se révéler des facteurs de succès de l'innovation managériale.

\subsection{Le manager de proximité et la diffusion de l'innovation managériale}

Toute innovation est caractérisée par sa diffusion (Rogers, 2010 ; Van de Ven et al., 2000), la capacité d'innovation d'une organisation dépend ainsi du processus de circulation de la connaissance (Nonaka, 1991). Cette diffusion et l'adoption des changements liés s'appuient sur un processus 
d'apprentissage (Argyris, 1986). Les recherches en sciences de gestion soutiennent que l'architecture cognitive de la connaissance /construction de la connaissance, enregistrement, transmission, reconstitution) conditionne le processus d'apprentissage organisationnel qui agit de manière rétroactive sur le processus d'innovation (Nonaka, 1991). Houle et al. (2015) présentent le manager comme un acteur clé du transfert, du partage et de la co-construction de connaissances lors du transfert des savoirs dans une démarche Lean. Dubouloz (2013) précise ses pratiques et ses champs d'intervention qui doivent soutenir la diffusion des nouvelles pratiques tout en accompagnant les modifications structurelles et interrelationnelles.

La littérature met en exergue des compétences, ou leviers, favorisant cette diffusion des connaissances et leur appropriation. Lors de l'implantation d'une innovation managériale, le manager dépasse sa fonction de gestionnaire. Il développe ainsi un leadership et mobilise des compétences en communication contribuant à la création des sens et établissant un lien entre les différents niveaux hiérarchiques. Nous développons ici les concepts de leadership et de communication en lien avec la création de sens.

\section{Le leadership}

Tremblay (2008) présente le leadership comme l'un des facteurs principaux de succès du processus d'innovation permettant d'encourager la participation du personnel, d'accompagner le changement et de mobiliser l'ensemble des acteurs. Northouse (2015, p. 124) définit le leadership comme le « processus par lequel une personne influence un groupe de personnes pour atteindre un objectif commun ». Cette influence peut se référer aux capacités mobilisatrices du manager évoquées par Barrand (2017, p. 125), c'està-dire un « catalyseur des énergies vers une finalité collective ». Le leader reconnaît les compétences de chacun et est capable d'amener chacun vers les objectifs fixés en associant management relationnel et opérationnel. II donne du sens aux activités réalisées et coordonne les opérationnels lors de l'adaptation de l'innovation en vue de son adoption. Le leadership, reconnu comme légitime, associé au temps et à l'apprentissage permet la mise en place d'une dynamique collaborative lors du processus d'innovation managériale.

Tremblay (2008) distingue deux types de leadership dans le cadre des innovations et du changement : le leadership d'un leader d'opinion et le leadership collectif. Le leader d'opinion, par sa relation de proximité avec les opérationnels contribue à la diffusion des nouvelles pratiques (Dopson et al., 2002 ; Flodgren et al., 2007). Il adopte un comportement positif favorisant le succès de l'innovation (Rogers, 2003 ; Dopson et al., 2002 ; Flodgren et al., 2007). Le leadership collectif, quant à lui, s'appuie sur l'association de compétences complémentaires et expertes au niveau du pilotage du projet et dans la gestion du changement (Tremblay, 2008). Il peut être contrarié par des rivalités entre les leaders, le manque de faisabilité du projet et la non considération de l'environnement. Au cours de leurs travaux portant sur le Lean Healthcare, Foropon et Landry (2014) révèlent l'importance du 
leadership combiné à une communication efficace communication afin d'obtenir l'adhésion et la mobilisation de l'ensemble des acteurs.

\section{La création de sens/la communication}

Desmarais et Abord de Chatillon (2010, p. 75) écrivent au sujet des managers qu'ils « créent du sens (sensemaking) et le communiquent à leur entourage (sensegiving) ». La création de sens se révèle ainsi dans la pratique discursive du manager (El Gaied, 2010). Lors du processus d'innovation, le manager doit mobiliser des compétences en communication (El Gaied, 2010 ; Olivesi, 2002). Une non-compréhension des enjeux et de l'intérêt de l'innovation peut nuire à sa diffusion (El Gaied, 2010). La mobilisation repose sur une stratégie établie et communiquée permettant aux acteurs de comprendre le sens donné à leurs actions (Fraisse et al., 2003). Fraisse et al., (2003, p. 157) écrivent que « les pratiques discursives deviennent ainsi un levier de changement sur lequel le manager peut agir ». Cependant, le discours doit intégrer le système de représentations des acteurs afin d'adapter la terminologie utilisée lors du processus d'apprentissage (Minvielle, 1999).

Cette littérature relative à l'importance du facteur managérial soulève une réflexion sur son adéquation avec la complexité du contexte hospitalier. Pour Alter (2010, p. 155), l'« innovation se heurte à l'organisation », une exploration du rôle managérial implique une prise en compte des caractéristiques des organisations de santé.

\subsection{Les établissements hospitaliers}

Denis et al., (2002) qualifient les établissements hospitaliers d'organisations pluralistes au regard de la diversité d'acteurs y exerçant, les décisions se négocient plus qu'elles ne s'imposent. La pluralité de culture, les intérêts divergents et le côtoiement de communautés de pratiques, définies comme « des lieux où se construisent en permanence des modèles locaux, des représentations partagées, des jargon » (Cohendet et al., 2003), renforcent la complexité structurelle des organisations de santé impactant ainsi le processus de diffusion des innovations.

Gheorghiu et Moatty (2013) présentent les trois pouvoirs de l'hôpital : médical, soignant et administratif. Si les médecins recrutent les patients, les directions soignante et administrative mettent à disposition les ressources. Cette interdépendance des pouvoirs et la coopération nécessaire, liée à la pluridisciplinarité et la transversalité des activités, ont provoqué une évolution du mode de gouvernance dont l'un des enjeux est la volonté de « décloisonner le fonctionnement de l'hôpital » (Fray, 2009, p. 156). Cependant, les intérêts divergents et les jeux de pourvoir, ont soulevé des résistances visà-vis de l'application de cette nouvelle gouvernance perçue comme « une révolution culturelle et structurelle modifiant les lignes hiérarchiques et fonctionnelles » (Fray, 2009, p. 156). Malgré les efforts entrepris la coopération est limitée au sein de l'hôpital qui ne parvient pas à construire le faire ensemble. Ces spécificités organisationnelles et l'environnement 
multi-professionnel peuvent entraver le processus d'innovation managériale (Ferlie et al., 2005 ; Kanter, 2006).

Le cadre théorique marque ainsi l'interdépendance entre les trois concepts explorés. Les innovations managériales, s'inscrivant au sein des spécificités contextuelles, sont influencées par le management, notamment le management de proximité et la capacité de mobilisation des compétences favorisant la diffusion des connaissances : le leadership et la communication.

\section{QUESTION DE RECHERCHE ET OBJECTIFS DE LA RECHERCHE}

Le constat d'une difficulté d'implantation des innovations managériales au sein des établissements hospitaliers et la mise en exergue d'une influence managériale sur le processus ont abouti à la question de recherche suivante :

\section{Comment le manager de proximité peut influencer la diffusion des connaissances relatives à l'innovation managériale au sein des établissements hospitaliers?}

Autrement dit, il s'agit de savoir comment le manager de proximité peut mobiliser les compétences nécessaires afin d'influencer le processus d'innovation managériale au sein des hôpitaux. Cet article apporte un éclairage sur la contribution du manager de proximité sur le processus d'innovation managériale au sein des établissements hospitaliers.

\section{CADRE MÉTHODOLOGIQUE ET EMPIRIQUE}

\subsection{Cadre méthodologique}

Afin de répondre à la question de recherche, nous souhaitons accéder au cœur du processus d'innovation managériale au plus près des acteurs concernés. Dans cet objectif, nous nous sommes orientés vers la recherche-intervention nous conduisant en immersion pendant une durée de six mois au sein d'une organisation et d'une culture propre permettant ainsi un apprentissage de son fonctionnement et une compréhension de ses codes. Merdinger- Rumpler et Nobre (2011, p. 55-56) décrivent l'intérêt de la recherche-intervention qui « permet une observation en profondeur 
et en temps réel des différents évènements et évolutions d'un phénomène organisationnel ». Le chercheur participe activement à la construction de la réalité, s'engage dans la concrétisation du réel, formalise le changement et « conçoit des outils qui aident à installer ce changement correspondant à ce modèle de gestion formalisé » (Gonzales-Laporte, 2014, p. 19). Les interactions entre le chercheur et les acteurs de l'organisation contribuent au processus de co-production de connaissances. L'intervention est initiée par l'organisation à partir d'une situation problématique peu compréhensible qui est ensuite formalisée (Aggeri, 2015).

Aggeri (2015, p. 5) présente les étapes de sa mise en œuvre :

- Une prise de connaissance du contexte permettant d'identifier le problème ;

- La mise en place d'un comité de pilotage se rencontrant régulièrement et constituant un espace d'échange entre chercheur et acteurs. La validation des connaissances se fait chemin faisant par les acteurs au cours du processus de création de connaissances ;

- Un raisonnement abductif visant à « générer des questions et hypothèses nouvelles à partir de l'observation de situations concrètes ».

Tout au long de notre immersion, nous accompagnons la mise en œuvre du Lean Healthcare et favorisons les interactions avec l'ensemble des acteurs de l'organisation tout en privilégiant les échanges avec les managers de proximité. Les données issues de nos échanges et observations sont recensées au sein d'un journal de bord documenté quotidiennement. L'analyse des données s'appuie sur une méthodologie en trois étapes (Krief et Zardet, 2013) :

- Une étape de pré-analyse consistant à une organisation des données ;

- Une étape de codage consistant à découper les données en unités, thèmes, sous-thèmes et idées clés afin de les associer à des catégories prédéfinies relatives au cadre conceptuel de notre recherche ;

- Une étape d'interprétation, synthèse permettant l'identification d'inférences.

Nous recourons à l'utilisation d'un logiciel d'analyse qualitative, Nvivo, permettant de « gérer mettre en forme et donner un sens aux données qualitatives » (Krief et Zardet, 2013, p.227) à partir d'une logique de « décontextualisation-recontextualisation du corpus » (Krief et Zardet, 2013, p.227) source de création de sens.

Ainsi, l'analyse des résultats empiriques est faite de manière abductive, par un aller-retour permanent entre le terrain et les concepts théoriques énoncés afin de conceptualiser la problématique et de tester des hypothèses. 


\subsection{Cadre empirique}

Notre terrain de recherche est le Centre Hospitalier de Luxembourg (CHL) situé au Grand-Duché de Luxembourg, Les activités du CHL, réparties dans quarante et un services cliniques, sont gérées conjointement par un cadre soignant-chef de service et un médecin-chef de service. L'organigramme met en évidence la politique médico-soignante soutenant la collaboration des médecins et des soignants. Avant d'envisager un déploiement plus large, la direction du CHL souhaite entreprendre une application de l'approche Lean Healthcare au sein d'un service pilote afin de résoudre les dysfonctionnements constatés. L'implantation du Lean Healthcare se déroule au sein du service de chimiothérapie ambulatoire caractérisé par une forte pluridisciplinarité mobilisant les compétences d'une équipe de médecins et de soignants spécialisés, collaborant avec le laboratoire et la pharmacie. La direction de l'établissement évoque des difficultés organisationnelles persistant depuis de nombreuses années et difficiles à résoudre en raison de la diversité des acteurs intervenant au niveau de la prise en charge des patients accueillis. Les retards au cours d'une prise en charge se répercutent sur les prises en charge suivantes. De plus, suite à l'arrivée de deux nouveaux médecins, l'activité a augmenté de $30 \%$ au cours des années 2016 et 2017.

Afin de répondre à la problématique posée relative au rôle du manager de proximité, nous axons notre recherche sur le rôle du cadre soignant du service de chimiothérapie ambulatoire assimilé à un manager de proximité.

\section{RÉSULTATS}

Les résultats sont présentés de manière chronologique selon les étapes successives de la mise en œuvre de l'innovation managériale.

\subsection{La prise de connaissance du terrain}

Notre immersion au sein du terrain débute en septembre 2017. La période d'observation permet d'appréhender le rôle et le vécu du manager de proximité. Nos investigations relatives au manager de proximité révèlent :

- De fortes exigences:

«On doit toujours rendre des comptes, on est évalué sur les statistiques du service et on n'a pas vraiment droit à l'erreur » (manager de proximité, service de chirurgiel. 
- Une faible latitude décisionnelle :

«C'est compliqué de prendre une décision. On doit d'abord la présenter à la directrice des soins qui valide ou pas. Elle nous demande de la justifier, il faut être convaincant. C'est difficile de faire valider une décision donc finalement ça limite l'envie de prendre des initiatives ou de proposer des choses » (manager de proximité, service de pédopsychiatrie) ;

"J'aimerai qu'on me fasse confiance, pouvoir décider et prendre des initiatives » (manager de proximité, service de chimiothérapie ambulatoire).

- Une collaboration médico-soignante vécue difficilement :

"C'est difficile de travailler avec les médecins, ils n'ont jamais le temps et ne comprennent pas nos difficultés et nos besoins. Ils sont juste axés sur leurs problèmes à eux » (manager de proximité, service de cardiologie).

- Des activités de gestion chronophages:

"J'ai plein de travail administratif, il y a le planning, les commandes de matériel, le contrôle des dossiers de soins » (manager de proximité, service de chirurgie orthopédique) ;

« Je suis débordé. Mes journées commencent à 6 h30 et terminent à 19h » (manager de proximité, service de chimiothérapie ambulatoire).

Cette période permet également de préciser les difficultés organisationnelles évoquées par la direction. En s'inscrivant dans une bureaucratie professionnelle, cloisonnée, le processus de prise en charge révèle des dysfonctionnements au niveau de ses interfaces, notamment des difficultés de coordination des acteurs (cf. figure 1).

Figure 1. Les interfaces du processus de prise en charge

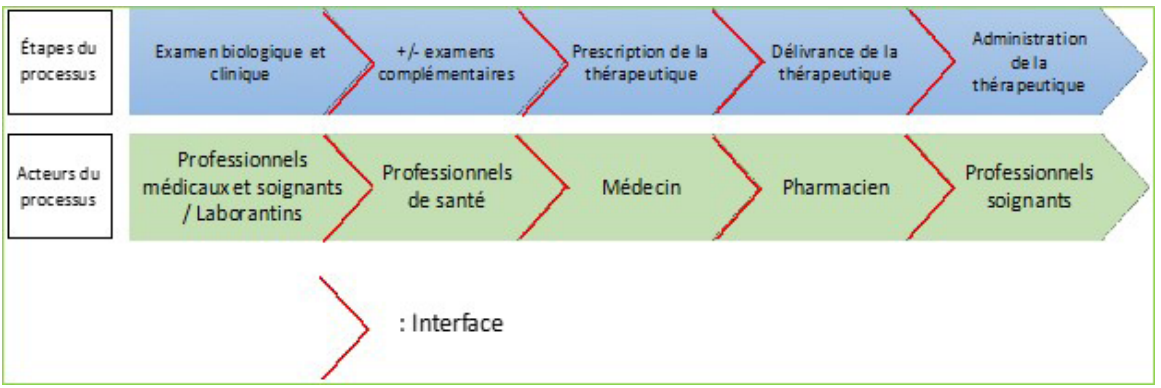

Notre intervention vise une optimisation des mécanismes de coordination à partir d'une approche collaborative intégrant l'ensemble des acteurs du processus. Nous retrouvons ici les principes du Lean Healthcare. 


\subsection{Le déploiement du Lean Healthcare}

Le déploiement de l'innovation managériale s'inscrit dans les trois étapes successives décrites précédemment (cf. paragraphe 2.1.).

\section{La phase de décision}

Si la décision d'implanter le Lean est entièrement portée et assumée par la direction, le manager de proximité intervient dans la diffusion des connaissances au cours de la phase de décision. Le comité de direction décide de la constitution d'un groupe de travail et propose au manager de proximité d'être le chef de projet qui doit dès lors suivre une formation visant l'acquisition des compétences requises à l'application de la méthodologie. Au cours de cette phase, le processus de diffusion des connaissances est initié par le manager de proximité à partir des compétences acquises. Il transmet son savoir aux acteurs impliqués dans le déploiement de l'innovation managériale, soient les membres du groupe de travail. Il utilise dans un premier temps de nombreux termes techniques et des acronymes renvoyant à la méthode et aux outils tels que «DMAIC » (Définir, Mesurer, Analyser, Innover, Contrôler) et «SIPOC » (Supplier, Input, Process, Output, Customer). Ses propos sont perçus comme très théoriques par les personnes présentes:

«C'est très théorique tout ça, c'est très abstrait » (médecin chef, service de chimiothérapie ambulatoire) ;

« Ça semble assez compliqué cette méthode, on a un peu de mal à comprendre où il veut en venir » (infirmier 1, service de chimiothérapie ambulatoire).

Suite aux réactions de ses interlocuteurs, il réajuste son discours et s'assure de s'exprimer en utilisant un langage précis tout en l'adaptant, que ce soit le contenu ou la forme. Lorsqu'il s'adresse aux médecins, il évoque les difficultés rencontrées par cette profession et précise l'intérêt de l'innovation au regard de ces difficultés. De la même façon, lorsqu'il explique l'intérêt de la méthode aux professionnels soignants, il fait référence à leurs difficultés :

"Je fais attention aux mots que j'utilise pour être le mieux compris possible » (manager de proximité, service de chimiothérapie ambulatoire) ;

«C'est une approche pluridisciplinaire (...) on va travailler ensemble sur le meilleur moyen d'améliorer la collaboration entre le service de chimiothérapie ambulatoire et la pharmacie » (manager de proximité s'adressant au pharmacien) ;

"Vous dépendez de la pharmacie et du laboratoire dans la prise en charge, alors il faut travailler avec eux pour améliorer les choses. Vous ne pouvez plus travailler seuls de votre côté » (manager de proximité s'adressant à un infirmier). 
Le manager adapte sa communication au contexte et au système de représentation des acteurs.

\section{La phase de mise en usage de l'innovation managériale}

La mise en usage du Lean Healthcare suit une méthodologie rigoureuse et structurée, suivant une démarche d'analyse des dysfonctionnements et de résolution de problèmes intitulée DMAIC (Define, Mesure, Analyse, Improve, Controll associée à une application de ses principes. Cinq réunions de travail sont ainsi planifiées sur une période de cinq mois à une fréquence régulière d'une réunion par mois, chacune correspondant à une étape du DMAIC. Le manager de proximité, nommé chef de projet, conduit les réunions et fait un retour à la directrice des soins après chaque réunion. Celle-ci déclare faire confiance au chef de projet et souhaite uniquement être informée de l'avancement de la démarche. Le manager de proximité s'attache à maintenir un rythme régulier afin de maintenir la dynamique de groupe. Suite à une indisponibilité répétée du médecin, il adapte à trois reprises le calendrier des réunions. Cependant la durée du projet lasse les participants :

«C'est quand même long, et ça prend du temps. C'est dur pour les collègues quand on doit s'absenter pour aller aux réunions » (infirmier 2, service de chimiothérapie ambulatoire) ;

" J'ai du mal à comprendre pourquoi il faut faire toute cette démarche, pour moi ce n'est pas compatible avec l'efficience » (médecin, service de chimiothérapie ambulatoire).

Malgré les critiques des participants au regard de certains outils permettant une analyse des difficultés rencontrées:

« On les connaît les problèmes pourquoi on doit faire tout ça ? " (Infirmier 3, service de chimiothérapie ambulatoire),

le manager de proximité fait preuve de ténacité et de persévérance :

«Ce n'est pas toujours facile, je sens qu'ils en ont marre, je dois faire beaucoup d'efforts pour maintenir le rythme » (manager de proximité, service de chimiothérapie ambulatoire).

Cette phase du processus marque une accélération de la diffusion des connaissances relatives à la méthodologie et à la philosophie Lean. Le manager de proximité transmet ses connaissances de manière plus précise aux membres du groupe projet mais également à d'autres acteurs le sollicitant s'interrogeant sur le projet en cours. Au cours de chacune des réunions, il accompagne les participants dans l'utilisation des outils Lean et leur transmet les modalités d'utilisation. 


\section{La phase de poursuite de l'usage de l'innovation managériale}

Les réunions de travail s'achèvent avec la définition de modifications organisationnelles décidées et initiées. Nous retournons ensuite ponctuellement sur le terrain pendant une période de six mois afin de recueillir les données relatives à la poursuite de l'usage de l'innovation managériale afin de recenser les données nécessaires à nos investigations :

«Les choses se sont beaucoup améliorées, il y a moins d'attente, on travaille plus facilement avec les autres services » (chef d'unité du département de biologie clinique) ;

«Finalement on a appris à résoudre nos problèmes en réfléchissant de manière plus poussée » (pharmacien).

Les acteurs ont modifié leur approche face aux problèmes en appliquant les connaissances relatives à la méthodologie Lean :

«On regarde régulièrement les chiffres sur les délais d'attente, ça nous permet d'avoir une idée précise de la situation » (infirmier 4, service de chimiothérapie ambulatoire).

Au cours de la poursuite de l'usage, la diffusion des connaissances est conditionnée par les résultats de la mise en usage de l'innovation. Les améliorations obtenues renforcent la légitimité et la reconnaissance du manager de proximité en tant qu'expert ou référent de l'innovation managériale, il est alors capable de diffuser les connaissances. Suite aux résultats de l'innovation managériale au sein du service de chimiothérapie, notamment la diminution des délais d'attente au cours de la prise en charge, de nombreux acteurs sollicitent le manager de proximité au sujet de la méthodologie Lean afin de de l'appliquer au sein de leurs services d'exercice.

Ces résultats contribuent à l'ouverture d'une discussion sur les résultats de la recherche.

\section{DISCUSSION}

\subsection{Discussion des résultats}

La diffusion des connaissances est présente tout au long du processus d'innovation managériale.

Les conditions soutenant l'influence du manager de proximité sont :

- Sa nomination en tant que chef de projet, soit sa légitimité, et sa maîtrise de l'innovation managériale ; 
- La pratique discursive : il intègre progressivement l'influence de son discours sur l'engagement des acteurs;

- Une connaissance de l'organisation et de ses acteurs lui donnant la capacité de défendre l'adéquation de l'innovation au terrain et aux difficultés rencontrées.

- La manager est cependant limité par des contraintes :

- Une faible latitude décisionnelle,

- Le manque de temps.

Au cours de l'étape de décision, la diffusion des connaissances est initiée à partir des savoirs acquis lors de la formation et s'appuie sur la pratique discursive. Comme le soulignent les travaux de Ring et Van de Ven (1994), la connaissance subit un changement cognitif et est modifiée suite à une interprétation par les récepteurs de l'information. Ainsi, les connaissances partagées par le manager de proximité peuvent soulever des incompréhensions et des critiques de la part des acteurs du fait d'une interprétation erronée suite à un discours très technique. Au terme de la phase de décision, malgré les réticences exprimées par les professionnels de terrain, le manager de proximité parvient à les convaincre à s'engager dans l'application du Lean Healthcare. En s'appuyant sur sa connaissance des acteurs, il développe progressivement son leadership grâce à une légitimité acquise par sa nomination en tant que chef de projet et sa maitrise de l'innovation managériale. Il combine ainsi le leadership d'un leader d'opinion et le leadership collectif soutenu par le management de projet associé. Au cours de l'étape de mise en usage, le manager de proximité poursuit cette transmission des connaissances grâce à une adaptation de la méthode au contexte et aux acteurs. La poursuite de l'usage est conditionnée par les résultats obtenus suite au déploiement de l'innovation managériales. Des améliorations organisationnelles vont favoriser la diffusion des connaissances au-delà du service de mise en œuvre.

\subsection{Les apports de la recherche}

L'apport principal de cette recherche porte sur les implications managériales. Par sa proximité avec les autres communautés de pratique et la diversité de ses relations interpersonnelles au sein de la bureaucratie professionnelle constituée par l'hôpital, il se positionne à la fois comme un intermédiaire vertical mais également un intermédiaire transversal. D’après Senge (2006), les managers, de tous les niveaux hiérarchiques ont une responsabilité sur l'instauration des conditions favorisant l'apprentissage soutenant le changement. Le manager doit être capable d'identifier les acteurs porteurs du changement et de les intégrer dans la diffusion du changement.

Sa hiérarchie doit cependant l'accompagner en lui permettant d'acquérir les compétences nécessaires : des aptitudes en communication et en management de projet. C'est ainsi qu'il pourra développer son leadership. Au-delà des activités de gestion indispensables au bon fonctionnement du 
service, la direction doit considérer ces compétences managériales afin de soutenir et favoriser toute démarche de transformation organisationnelle.

\subsection{Les limites de la recherche}

Les résultats de notre recherche sont issus de l'implantation d'une innovation managériale précise, le Lean Healthcare, ce qui constitue une limite de notre recherche. De même, les spécificités du terrain de recherche et les conditions des établissements hospitaliers luxembourgeois disposant de ressources suffisantes peuvent influencer les résultats de la recherche. Une réplication de cette recherche implique de considérer les conditions contextuelles intégrant la disponibilité des ressources nécessaires, notamment le temps mis à disposition du déploiement de l'innovation managériale.

\subsection{Les perspectives de recherche}

Les établissements hospitaliers, assimilés à des anarchies organisées (Cohen et al., 1991) sont caractérisés par des interactions complexes orientées par le mode de gouvernance, le système de prise de décision et les rapports de pouvoir. De plus, des paramètres sociologiques tels que la distance sociale (Gheorghiu et Moatty, 2013), induite par la diversité de catégories professionnelles, peuvent entraver les interactions et la diffusion des innovations managériales. Il devient nécessaire de mettre en place des interactions complexes, telles que des acteurs ou des étapes intermédiaires, favorisant la transmission et le partage des connaissances. Une perspective de recherche s'ouvre sur la mise en place de ces interactions au sein de cette complexité structurelle afin de favoriser la diffusion et l'adoption de l'innovation managériale. En effet, au-delà des aspects structurels, la complexité des organisations existe à travers les interactions entre les acteurs, et également les interactions entre les acteurs et les outils mobilisés (Schmitt, 2020). Une telle recherche implique d'intégrer le concept de coopération à l'hôpital, souhaitée ou contrainte. En effet, « les engagements dans le collectif restent instables s'ils ne s'inscrivent pas dans la durée, car les relations de coopération au travail ne sont pas réductibles à des formes d'interaction » (Gheorghiu et Moatty, 2013, p. 38).

\section{CONCLUSION GÉNÉRALE}

Notre recherche révèle la capacité du manager de proximité à devenir un vecteur de la diffusion des connaissances au sein de la complexité des hôpitaux. Cette habilitation est cependant conditionnée par la mobilisation de leviers managériaux ou compétences : le leadership, la communication et le management de projet. De même, des facteurs organisationnels tels qu'une évolution de son pouvoir décisionnel et la mise en place d'interactions avec 
d'autres acteurs de l'organisation peuvent favoriser son influence. Notre travail de recherche soulève l'importance de l'approche collaborative à l'hôpital en incluant l'ensemble des acteurs et en les rassemblant dans la réflexion portant sur les difficultés organisationnelles. La résolution des difficultés implique la contribution de chaque acteur de l'institution de manière égale en considérant le gisement d'amélioration issu des professionnels de terrain.

Il est cependant à noter les limites et les perspectives de cette recherche. Des limites émergent de la méthodologie appliquée et des spécificités contextuelles. Une réplication de la recherche menée contribuera à une généralisation de ses résultats. De plus, les caractéristiques de notre terrain, les organisations de santé, ouvrent des perspectives de recherche portant sur les interactions professionnelles favorisant la diffusion des innovations managériales au sein de cette complexité structurelle.

\section{RÉFÉRENCES}

Aggeri, F. (2015). La recherche-intervention : fondements et pratiques. À la pointe du management. Ce que la recherche apporte au manager. Paris : Dunod.

Alter, N. (2010). L'innovation ordinaire. Paris : Presses universitaires de France.

Aoki, M. (1988). Information, incentives and bargaining in the Japanese economy: A microtheory of the Japanese Economy. Cambdrige: Cambridge University Press.

Argyris, C. (1986). Reinforcing organizational defensive routines: An unintended human resources activity. Human Resource Management, 25(4), 541-555.

Armbruster, H., Bikfalvi, A., Kinkel, S., \& Lay, G. (2008). Organizational innovation: The challenge of measuring non-technical innovation in large-scale surveys. Technovation, 28(10), 644-657.

Balogun, J. (2010). Comment les managers intermédiaires négocient-ils le changement?. Conduite du changement : concepts-clés : 50 ans de pratiques issues des travaux de 25 grands auteurs, 73.

Barrand, J. (2017). Le manager agile : Agir autrement pour la survie des entreprises. $3^{\mathrm{e}}$ édition. Paris : Dunod.

Belorgey, N. (2010). L'hôpital sous pression : Enquête sur le "nouveau management public ». Paris : La Decouverte.

Bentahar, O., \& Benzidia, S. (2019). Supply chain management de la santé. Éditions EMS.

Birkinshaw, J., Hamel, G., \& Mol, M. J. (2008). Management innovation. Academy of Management Review, 33(4), 825-845.

Cohen, M. D., March, J. G., \& Olsen, J. P. (1991). Le modèle du « garbage can » dans les anarchies organisées. Décisions et Organisations, 163-204.

Cohendet, P., Créplet, F., \& Dupouët, O. (2003). Innovation organisationnelle, communautés de pratique et communautés épistémiques : Le cas de Linux. Revue Française de Gestion, 5 , 99-121.

Damanpour, F. (1991). Organizational innovation: A meta-analysis of effects of determinants and moderators. Academy of Management Journal, 34(3), 555-590. 
Deblois, S., \& Lepanto, L. (2013). Implantation et effets du Lean et du six sigma en milieu de soins aigus. Montréal: Direction de l'évaluation des technologies et des modes d'intervention en santé (DETMIS) - Centre hospitalier de l'Université de Montréal.

Denis, J. L., Hébert, Y., Langley, A., Lozeau, D., \& Trottier, L. H. (2002). Explaining diffusion patterns for complex health care innovations. Health Care Management Review, 27(3), 60-73.

Desmarais, C., \& Abord de Chatillon, E. (2010). Le rôle de traduction du manager : Entre allégeance et résistance. Revue Française de Gestion, 205(6), 71-88.

Dopson, S., FitzGerald, L., Ferlie, E., Gabbay, J., \& Locock, L. (2002). No magic targets! Changing clinical practice to become more evidence based. Health Care Management Review, 27(3), 35-47.

Dubouloz, S. (2013). Les barrières à l'innovation organisationnelle : Le cas du Lean Management. International Management, 17(4), 121-144.

Dubouloz, S. (2014). Innovation organisationnelle et pratiques de mobilisation des RH. Revue Française de Gestion, 1, 59-85.

El Gaied, M., (2010). La transplantation managériale de l'hôpital : Démarche qualité, communication symbolique et changement des mentalités au travail. Sarrebruck : Éditions Universitaires Européennes.

Ferlie, E., Fitzgerald, L., Wood, M., \& Hawkins, C. (2005). The non-spread of innovations: The mediating role of professionals. Academy of Management Journal, 48(1), 117-134.

Flodgren, G., O'Brien, M. A., Parmelli, E., \& Grimshaw, J. M. (2007). Local opinion leaders: Effects on professional practice and healthcare outcomes. Cochrane Database of Systematic Reviews, (6).

Foropon, C., \& Landry, S. (2014). Le déploiement du Lean à l'hôpital Saint-Boniface : L'importance du leadership transformationnel. Gestion, 39(3), 107-115.

Fraisse, S., Robelet, M., \& Vinot, D. (2003). La qualité à l'hôpital : Entre incantations managériales et traductions professionnelles. Revue Française de Gestion, 5, 155-166.

Fray, A. (2009). Nouvelles pratiques de gouvernance dans le milieu hospitalier : Conséquences managériales sur les acteurs. Management \& Avenir, 28(8), 142-159.

Gheorghiu, M. D., \& Moatty, F. (2013). L'hôpital en mouvement. Changements organisationnels et conditions de travail. Paris : Liaisons Editions.

Gonzalez-Laporte, C. (2014). Recherche-action participative, collaborative, intervention... Quelles explicitations? Thèse de doctorat, Labex ITEM.

Hamel, G. (2006). The why, what, and how of management innovation. Harvard Business Review, $84(2), 72$.

Hasle, P. (2014). Lean production - An evaluation of the possibilities for an employee supportive Lean practice. Human Factors and Ergonomics in Manufacturing \& Service Industries, 24(1), 40-53.

Houle, L., Bareil, C., Gosselin, A., \& Jobin, M. (2015). Le déploiement du lean santé au Québec en mode agile. Question(s) de Management, 10(2), 45-64.

Kanter, R. M. (1982). The middle manager as innovator. Harvard business review, 60(4), 95-105.

Kanter, R. M. (2006). Innovation: The classic traps. Harvard Business Review, 84(11), 72-83.

Kim, C. S., Spahlinger, D. A., Kin, J. M., \& Billi, J. E. (2006). Lean health care: What can hospitals learn from a world-class automaker?. Journal of Hospital Medicine: An Official Publication of the Society of Hospital Medicine, 1(3), 191-199.

Krief, N., \& Zardet, V. (2013). Analyse de données qualitatives et recherche-intervention. Recherches en Sciences de Gestion, 95(2), 211-237.

Lozeau, D. (2010). Le difficile ancrage de la gestion de la qualité dans les hôpitaux publics: écarts et cohabitation entre trois archétypes organisationnels. Nouvelles Pratiques Sociales, 22(2), 66-82. 
Menard, C. (1995), La nature de l'innovation organisationnelle : éléments de réflexion Revue d'économie industrielle, numéro exceptionnel « Economie industrielle : développements récents ». 173-192.

Merdinger-Rumpler, C., \& Nobre, T. (2011). Quelles étapes pour la conduite du changement à l'hôpital ?. Gestion 2000, 28(3), 51-66.

Minvielle, E., \& Durand, V. (2016). À la découverte des innovations managériales hospitalières (No. hal-01677506).

Minvielle, E. (1999). Les politiques d'amélioration de la qualité des soins à l'hôpital. Quel fondement organisationnel ?. Politiques et Management Public, 17(4), 59-84.

Nicolay, C. R., Purkayastha, S., Greenhalgh, A., Benn, J., Chaturvedi, S., Phillips, N., \& Darzi, A. (2012). Systematic review of the application of quality improvement methodologies from the manufacturing industry to surgical healthcare. British Journal of Surgery, 99(3), 324-335.

Niosi, J., Bellon, B., Saviotti, P., \& Crow, M. (1992). Les systèmes nationaux d'innovation : A la recherche d'un concept utilisable. Revue Française d'Economie, 7(1), 215-250.

Nonaka, I. (1991). The Knowledge-Creating Company, Managing for the long term. Harvard Business Review, 162-171.

Northouse, P. G. (2018). Leadership: Theory and practice. New York, NY: Sage Publications.

Ohno, T. (1988). Toyota production system : Beyond large-scale production. Boca Raton : CRC Press.

Olivesi, S. (2002). La communication au travail. Une critique des nouvelles formes de pouvoir dans les entreprises. Grenoble: Presses universitaires de Grenoble.

Radnor, Z. J., Holweg, M., \& Waring, J. (2012). Lean in healthcare: The unfilled promise?. Social Science and Medicine, 74(3), 364-371.

Ring, P. S., \& Van de Ven, A. H. (1994). Developmental processes of cooperative interorganizational relationships. Academy of Management Review, 19(1), 90-118.

Rogers, E. M. (2010). Diffusion of innovations. New York, NY : Simon and Schuster.

Schmitt, C. (2020). Des nouveaux enjeux de la complexité. Projectics / Proyéctica / Projectique, 25(1), 5-6.

Scozzi, B., Garavelli, C., \& Crowston, K. (2005). Methods for modeling and supporting innovation processes in SMEs. European Journal of Innovation Management, 8(1), 120-137.

Senge, P. M., Gauthier, A., \& Plagnol, H. (2006). La cinquième discipline (Vol. 175). Paris: First.

Tremblay, D. (2008). La traduction d'une innovation organisationnelle dans les pratiques professionnelles de réseau : l'infirmière pivot en oncologie. Montréal : Université de Montréal.

Van de Ven, A. H., Angle, H. L., \& Poole, M. S. (2000). Research on the management of innovation: The Minnesota studies. Oxford: Oxford University Press.

Womack, J. P., Jones, D. T., \& Roos, D. (2007). The machine that changed the world: The story of lean production--Toyota's secret weapon in the global car wars that is now revolutionizing world industry. Simon and Schuster.

Noura Zaghmouri est Maître de conférences en Sciences de gestion à l'Université de Reims Champagne-Ardenne. Après une expérience professionnelle en tant que manager au sein des hôpitaux, elle s'engage dans une thèse de Doctorat ayant pour sujet principal le lean healthcare. Ses travaux de recherche portent sur le management et les innovations managériales au sein de la complexité organisationnelle des hôpitaux. 